Case Report

\title{
Aortic Root Abscess with Aorta to Right Atrium Fistula due to Vancomycin-Intermediate Staphylococcus aureus (VISA)
}

\author{
Stephen J. Hankinson (D), Elliot A. Sultanik $(\mathbb{D}$, and Gautam V. Ramani $(\mathbb{D}$ \\ Department of Medicine, Division of Cardiovascular Medicine, University of Maryland School of Medicine, Baltimore, \\ Maryland 21201, USA \\ Correspondence should be addressed to Stephen J. Hankinson; shankinson@som.umaryland.edu
}

Received 2 March 2019; Revised 1 May 2019; Accepted 7 May 2019; Published 29 May 2019

Academic Editor: Antonio de Padua Mansur

Copyright (C) 2019 Stephen J. Hankinson et al. This is an open access article distributed under the Creative Commons Attribution License, which permits unrestricted use, distribution, and reproduction in any medium, provided the original work is properly cited.

\begin{abstract}
We present a case of root abscess with aorta to right atrium fistula due to vancomycin-intermediate Staphylococcus aureus (VISA) after limb amputation and cardiac surgery. Patient underwent redo aortic valve replacement, patch repair of aorta to right atrial fistula, and tricuspid valve repair with a ring. Fistula formation is a rare complication of prosthetic valve endocarditis (PVE). This is the first case to discuss aortocavitary fistula (ACF) formation due to VISA. Transesophageal echocardiogram (TEE) is the preferred imaging modality to diagnose ACF.
\end{abstract}

\section{Introduction}

The emergence of multidrug-resistant (MDR) organisms makes the medical management of endocarditis increasingly difficult. TEE is the preferred imaging modality to diagnose ACF. Surgical repair continues to be the definitive treatment option for ACF.

\section{Case Presentation}

Our patient is a 74-year-old man with a history of rheumatoid arthritis (on prednisone), left below the knee amputation (BKA), coronary artery bypass graft (CABG) with a saphenous vein graft to the right coronary artery (SVG to RCA), aortic valve replacement (AVR) with a bioprosthetic valve, and mitral valve replacement (MVR) with a bioprosthetic valve who presented with fever, chills, and generalized weakness after a prolonged course of vancomycin for methicillinresistant Staphylococcus aureus (MRSA) bacteremia.

The patient was previously admitted for left foot MRSA osteomyelitis. During that hospitalization, the patient had a myocardial infarction. Source control obtained with BKA of the left leg and five days later subsequently underwent CABG (SVG to RCA), AVR for severe aortic stenosis (AS), and
MVR for severe mitral regurgitation (MR). The patient was discharged with a six-week course of vancomycin; however, vancomycin course was extended due to BKA wound that required skin graft surgery. Eighty-eight days after cardiac surgery, the patient was readmitted for MRSA bacteremia attributed to cellulitis of the BKA stump vs. endocarditis. Transthoracic echocardiogram (TTE) at that time was negative for PVE. Patient was treated with a six-week course of vancomycin, and BKA stump cellulitis subsequently resolved. Five days after completing antibiotics, the patient presented with MRSA bacteremia, which progressed to VISA bacteremia with a vancomycin minimum inhibitory concentration (MIC) of $4 \mu \mathrm{g} / \mathrm{mL}$ (Table 1). The patient was started on daptomycin, ceftaroline, and rifampin for VISA bacteremia. TEE showed aortic root thickening suggestive of abscess with associated structural defect, a mobile $2.4 \mathrm{~cm} \times 1.9 \mathrm{~cm}$ echodensity projecting into the right atrium (RA) (Figure 1(a)), and left to right shunting (Figure 1(b)).

The patient subsequently underwent cardiac surgery for redo AVR with a bovine pericardial tissue valve, patch repair of aorta to right atrial fistula with a bovine pericardial patching material from both the left ventricular side and right atrial side, and tricuspid valve repair with a ring. Postsurgery TTE demonstrated a normal left ventricle size and systolic 
TABLE 1: Culture data during protracted use of vancomycin.

\begin{tabular}{lccc}
\hline Type of culture & Date & Organism & $\begin{array}{c}\text { Vancomycin MIC } \\
(\mu \mathrm{g} / \mathrm{mL})\end{array}$ \\
\hline Left foot wound & $11 / 10 / 2017$ & MRSA & $\leq 0.5$ \\
Blood & $2 / 27 / 2018$ & MRSA & 1 \\
Blood & $4 / 16 / 2018$ & MRSA & 2 \\
Blood & $4 / 26 / 2018$ & MRSA & 4 \\
\hline
\end{tabular}

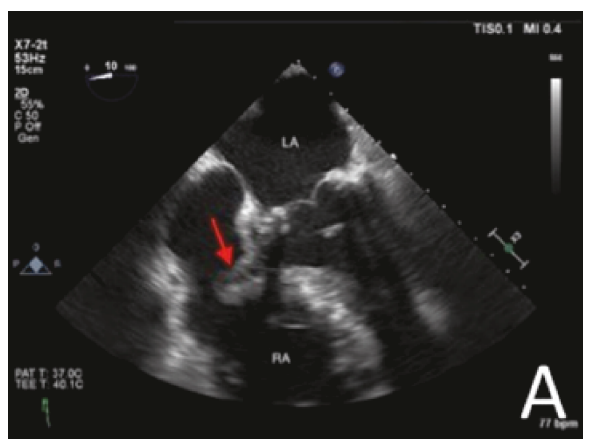

(a)

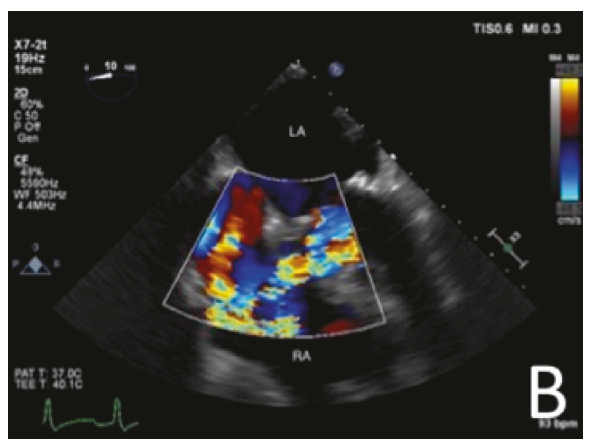

(b)

Figure 1: TEE demonstrates mobile echodensity (red arrow) measuring $2.4 \mathrm{~cm} \times 1.9 \mathrm{~cm}$ in the RA associated with the wall of the atrium at the site of the suspected defect (a). TEE demonstrates atrioventricular defect and a systolic left to right shunt suggestive of aortic root abscess and erosion into the RA (b).

function, a normal right ventricle size and function, a bioprosthetic valve in aortic position (mean gradient $20 \mathrm{mmHg}$ and peak gradient $45 \mathrm{mmHg}$ ) and no aortic regurgitation, and an annuloplasty ring indicative of tricuspid valve repair.

\section{Discussion}

This case discusses ACF formation as a complication of cardiac surgery shortly after limb amputation. PVE is a devastating complication of valve replacement with the prevalence being $4.1 \%$ at 4 years after primary valve replacement with the greatest risk occurring 1 to 2 months after surgery [1].

$\mathrm{ACF}$ is a rare condition caused by congenital abnormalities, aortic dissection, valve replacement, and infective endocarditis (IE) [2]. The pathogenesis of ACF related to infection is caused by extension and infiltration of abscesses related to endocarditis. Annular infection affects contiguous tissue leading to pyogenesis and tissue necrosis, which causes formation of an abscess cavity. As a result, the weakened necrotic myocardium may expand and rupture which may create intracardiac fistulous communications or even pericardial shunts [3]. Echocardiography can be used to identify the location of the lesion while angiography is often used to demonstrate the course of the lesion and define the surgical approach. Anguera et al. demonstrated that the detection of ACF for TTE and TEE is $53 \%$ and $97 \%$, respectively [4]. TEE is the superior imaging technique because the flow across the fistula is highly turbulent and Doppler mapping can easily detect pressure differences between the aorta and cardiac chambers even when the fistulous orifice is small; therefore, TEE allows for the detection of almost all fistulas and allows the optimal characterization of each fistula tract. Additionally, TEE is superior to TTE in assessing valve function and morphology as well as delineating intracardiac pathology such as complications of endocarditis, namely, root abscess and fistulas. Furthermore, TEE has a better signal to the noise ratio and proximity of the transducer to the heart leading to higher quality images with lesser attenuation [5].

Our case is unique because it is the first case of ACF due to VISA. Our patient had extensive comorbidities prior to BKA including cardiac issues, sepsis, and steroid use, all of which have been shown to be predictors of increased mortality and postoperative complications within 30 days after BKA [6]. Given these comorbidities, perhaps a longer time course should have elapsed between the BKA in order to further optimize the patient prior to cardiac surgery. Overall, these comorbidities lead to a prolonged course of vancomycin, which resulted in the development of a MDR organism.

VISA and vancomycin-resistant S. aureus (VRSA) are relatively rare infections that occur in the setting of heavy prior use of glycopeptide antibiotics [7]. The Clinical Laboratory Standards Institute (CLSI) defines VISA as a vancomycin MIC of $4-8 \mu \mathrm{g} / \mathrm{mL}$ and VRSA as a vancomycin MIC of $\geq 16 \mu \mathrm{g} / \mathrm{mL}$ [8]. Vancomycin $\mathrm{MIC} \geq 4 \mu \mathrm{g} / \mathrm{mL}$ is associated with vancomycin treatment failures in MRSA IE, which is a novel concept for this cohort [9]. MRSA resistance to vancomycin occurs through a variety of mechanisms including cell wall thickening, decreased autolysis, reduced production of cell surface protein A, increased capsule expressions, increased D-alanylation of teichoic acids, and reduced agr activity [8]. Given the prolonged course of vancomycin prior to the discovery of the ACF, it is difficult to identify whether the valve annulus was seeded during implantation of the valve or BKA stump cellulitis lead to hematogenous seeding of the valve.

\section{Conclusion}

Presented above was a case of a patient with an ACF due to VISA. This case demonstrated that protracted use of vancomycin resulted in VISA IE. Clinicians should exercise caution in patients with high bacterial load infections after prolonged antibiotic use. Infectious disease specialist 
consultation, change in antibiotic regimen, and aggressive surgical management are appropriate for the management of VISA IE.

\section{Conflicts of Interest}

The authors declare that they have no conflicts of interest.

\section{Supplementary Materials}

"Color doppler video" (supplementary material 1) $2.4 \mathrm{~cm} \times$ $1.9 \mathrm{~cm}$ mobile echodensity in the RA with a systolic left to right shunt. (Supplementary Materials)

\section{References}

[1] J. F. Sabik, B. W. Lytle, E. H. Blackstone, A. G. M. Marullo, G. B. Pettersson, and D. M. Cosgrove, "Aortic root replacement with cryopreserved allograft for prosthetic valve endocarditis," The Annals of Thoracic Surgery, vol. 74, no. 3, pp. 650-659, 2002, discussion 659.

[2] E. A. Fierro, R. R. Sikachi, A. Agrawal, I. Verma, M. Ojrzanowski, and S. Sahni, "Aorto-atrial fistulas: a contemporary review," Cardiology in Review, vol. 26, no. 3, pp. 137144, 2018.

[3] N. Kang, S. Wan, C. S. Ng, and M. J. Underwood, "Periannular extension of infective endocarditis," Annals of thoracic and cardiovascular surgery: official journal of the Association of Thoracic and Cardiovascular Surgeons of Asia, vol. 15, no. 2, pp. 74-81, 2009.

[4] I. Anguera, J. M. Miro, I. Vilacosta et al., "Aorto-cavitary fistulous tract formation in infective endocarditis: clinical and echocardiographic features of 76 cases and risk factors for mortality," European Heart Journal, vol. 26, no. 3, pp. 288297, 2005.

[5] K. Ananthasubramaniam, "Clinical and echocardiographic features of aorto-atrial fistulas," Cardiovascular Ultrasound, vol. 3, no. 1,2005 .

[6] P. J. Belmont Jr., S. Davey, J. D. Orr, L. M. Ochoa, J. O. Bader, and A. J. Schoenfeld, "Risk factors for 30-day postoperative complications and mortality after below-knee amputation: a study of 2,911 patients from the national surgical quality improvement program," Journal of the American College of Surgeons, vol. 213, no. 3, pp. 370-378, 2011.

[7] G. Chesi, A. Colli, C. A. Mestres, G. Gambarati, F. Boni, and T. Gherli, "Multiresistant-MRSA tricuspid valve infective endocarditis with ancient osteomyelitis locus," BMC Infectious Diseases, vol. 6, no. 1, 2006.

[8] B. P. Howden, J. K. Davies, P. D. Johnson, T. P. Stinear, and M. L. Grayson, "Reduced vancomycin susceptibility in Staphylococcus aureus, including vancomycin-intermediate and heterogeneous vancomycin-intermediate strains: resistance mechanisms, laboratory detection, and clinical implications," Clinical Microbiology Reviews, vol. 23, no. 1, pp. 99-139, 2010.

[9] A. M. Casapao, S. L. Davis, J. P. McRoberts et al., "Evaluation of vancomycin population susceptibility analysis profile as a predictor of outcomes for patients with infective endocarditis due to methicillin-resistant Staphylococcus aureus," Antimicrobial Agents and Chemotherapy, vol. 58, no. 8, pp. 4636-4641, 2014. 


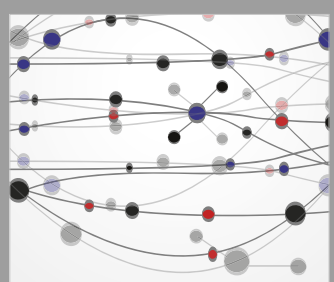

The Scientific World Journal
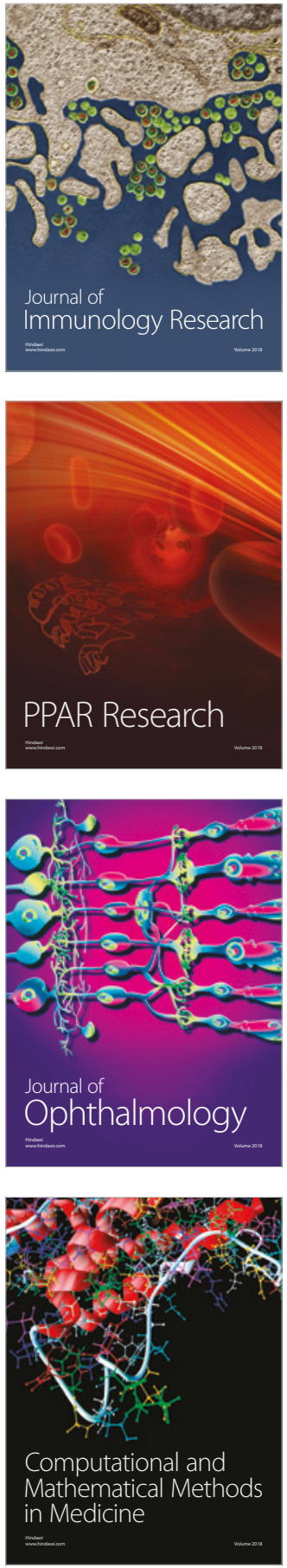

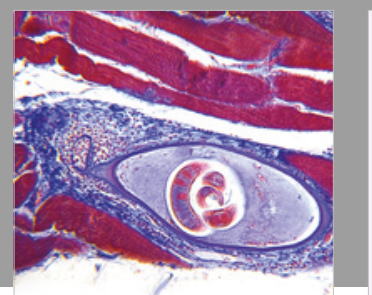

Gastroenterology Research and Practice

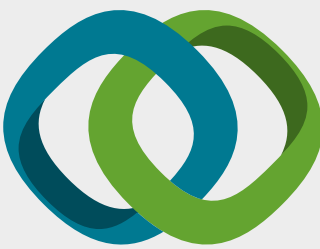

\section{Hindawi}

Submit your manuscripts at

www.hindawi.com
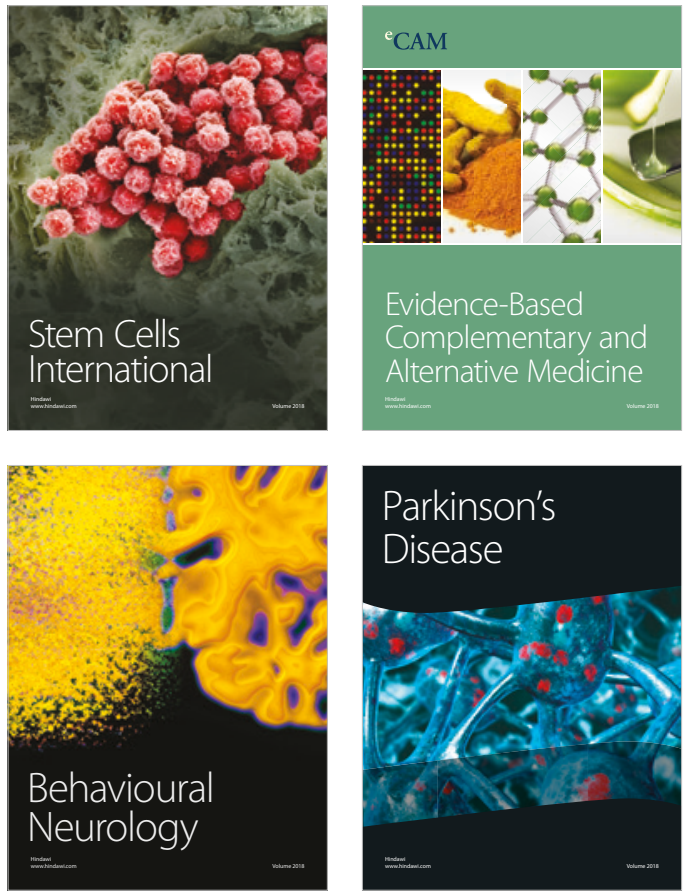

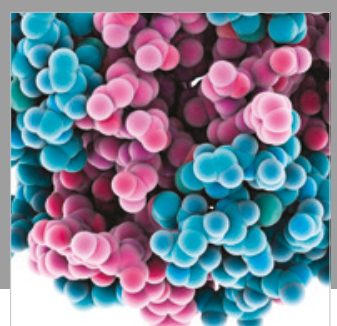

ournal of

Diabetes Research

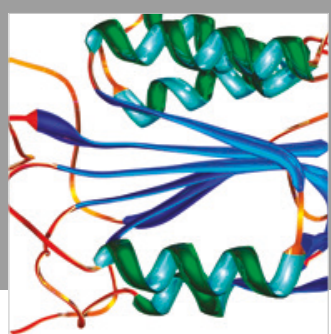

Disease Markers
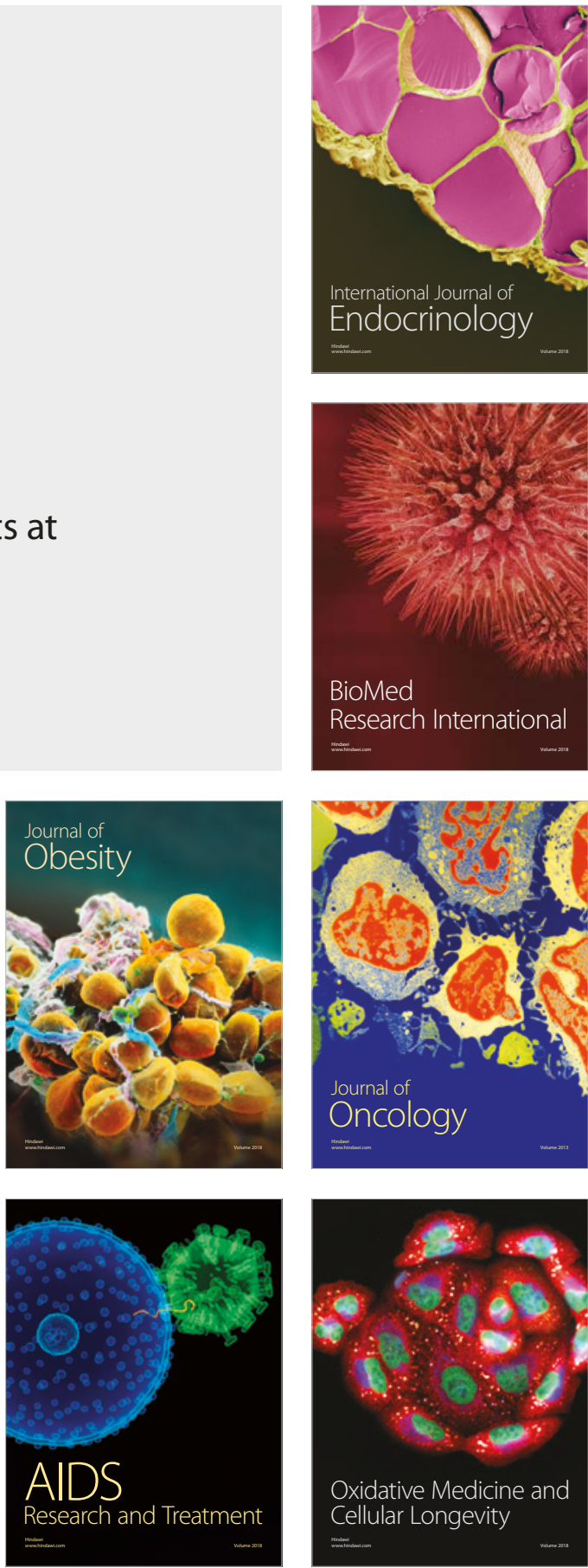